\title{
Vertical Toxoplasmosis in a Murine Model. Protection after Immunization with Antigens of Toxoplasma gondii Incorporated into Liposomes
}

\author{
Mohamed MA Elsaid, Maria S Martins, Frédéric Frézard*, Erika M Braga, \\ Ricardo WA Vitor/ +
}

Departamento de Parasitologia *Departamento de Fisiologia e Biofísica, Instituto de Ciências Biológicas, Universidade Federal de Minas Gerais, Caixa Postal 486, 31270-901 Belo Horizonte, MG, Brasil

Distinct Toxoplasma gondii antigens were entrapped within liposomes and evaluated for their ability to protect Balb/c mice against congenital transmission: soluble tachyzoite antigen (L/STAg), soluble tissue cyst antigen (L/SCAg), soluble tachyzoite plus tissue cyst (L/STCAg) or purified 32kDa antigen of tachyzoite $(L / p T A g)$. Soluble tachyzoite antigen alone in PBS (STAg) or emulsified in Freund's Complete Adjuvant (FCA/STAg) was also evaluated. Dams were inoculated subcutaneously with these antigens 6, 4 and 2 weeks prior to a challenge with four tissue cysts of the P strain of T. gondii orally between 10 and 14 days of pregnancy. Significant diminution differences were observed between the frequency of infected pups born of the dams immunized with the antigens incorporated into liposomes and that of pups born of the dams immunized with antigen emulsified in FCA or non immunized group $(p<0.05)$. There was a significant decrease in the number of pups born dead in the groups L/STAg, L/SCAg and L/pTAg when compared with pups from all other groups ( $p<0.05)$. All dams immunized with or without adjuvant showed an antibody response and a proliferation of T-cells. However, no correlation was found between immune response and protection against the challenge.

Key words: Toxoplasma - immunoprotection - congenital transmission - mice - liposomes

Toxoplasma gondii causes severe damage to the central nervous system in immunosuppressed individuals, particularly Aids patients (Luft et al. 1993) and in cases of congenital transmission (Wong \& Remington 1994). When women or sheep are infected for the first time during pregnancy, the maternal immune response seems to protect the mother but not the fetus (Remington et al. 1994). Previous studies have shown that cellular immunity is depressed during pregnancy (Holland et al. 1984) but recent studies have shown that, rather than a depression of the immune response, a predominant Type 2-like response may explain the maternal fetal tolerance (Wegmann et al. 1993, Thouvenin et al. 1997). Immunity induced by a previous infection in humans or sheep is effective in the prevention of

This study was supported by Fundação de Amparo à Pesquisa do Estado de Minas Gerais, Programa de Apoio aos Núcleos de Excelência and Conselho Nacional de Desenvolvimento Científico e Tecnológico.

${ }^{+}$Corresponding author. Fax: +55-31-499.2970. E-mail: vitorrwa@icb.ufmg.br

Received 10 April 2000

Accepted 9 August 2000 congenital transmission and parasite-induced abortion (Beverley \& Watson 1971).

Roberts and Alexander (1992) demonstrated that the Balb/c mouse can be used as a model of human or ovine congenital $T$. gondii infection. Infection of dams for the first time during the early period of gestation (day 7) results in fetal death, reabsorption or still-birth. On the other hand, mortality is reduced if dams are infected later in pregnancy (day 12). Neverthless, Balb/c dams are infected several weeks before mating, they develop an immunity capable to protect totally their embryos, even if reinfection occurs during pregnancy. This model is suitable for testing new chemotherapeutic agents to prevent the vertical transmission of $T$. gondii during acute phase (Fux et al. 2000).

Vaccination with live attenuated $T$. gondii in mice has been found to limit abortion and vertical disease transmission during pregnancy (McLeod et al. 1988). Furthermore, vaccination with soluble tachyzoite antigen (STAg) incorporated into liposomes prevented fetal death, and congenital infection was significantly curtailed (Roberts et al. 1994). However, the development of vaccines in the future will need to take into account all the lifecycle stages to be targeted, as well as the type of immune responses that must be generated in each tissue site. 
In a number of studies, Freund's complete adjuvant has been shown to be ineffective in enhancing protection against toxoplasmosis (Kasper et al. 1986, Alexander et al. 1993). However, administration of purified $T$. gondii antigen in the encapsulated form in liposomes (Bulow \& Boothroyd 1991, Elsaid et al. 1999) or in non-ionic surfactant vesicles (Alexander et al. 1993), was found to enhance protection against infection by $T$. gondii in mice. Furthermore, immunization with Escherichia coli expressed recombinant SAG1 (Petersen et al. 1998) or with STAg together with cytokine interleukin-12 (Yap et al. 1998), induced a partial protection against lethal infection with $T$. gondii in mice.

In this study, we determined the ability of different stage-specific soluble antigens of $T$. gondii and of a mixture of them in the encapsulated form in liposomes to reduce congenital transmission of toxoplasmosis in Balb/c mice.

\section{MATERIALS AND METHODS}

Mice - Three to four months-old female Balb/c mice were obtained from the experimental animal breeding laboratory of the Universidade Federal de Minas Gerais, divided in nine groups of seven animals.

Parasites - Tissue cysts were obtained from the brain of Swiss mice infected two months previously with $T$. gondii $\mathrm{P}$ strain of low virulence or moderately virulent $\mathrm{C} 4$ strain, isolated from dogs in São Paulo, Brazil (Jamra \& Vieira 1991). For antigen preparation, tissue cysts from the brain tissue of mice infected with the $T$. gondii $\mathrm{C} 4$ strain were purified and processed as described in our previous study (Elsaid et al. 1999). Tissue cysts of the $P$ strain were used for oral-challenge infection of immunized mice. Tissue cysts of the low virulence $\mathrm{P}$ strain were used for oral challenge since normal dams infected with four cysts survive until delivery ( $0 \%$ mortality at day 30 after inoculation).
The N strain (Nobrega et al. 1952) was used to produce the STAg and the purified tachyzoite antigen (pTAg).

Tachyzoite antigen - STAg and pTAg were obtained as previously described (Elsaid et al. 1999). Briefly, tachyzoites of the $\mathrm{N}$ strain were sonicated using five periods of $30 \mathrm{sec}$ at $40 \mathrm{hertz}$ at $1 \mathrm{~min}$ intervals. After centrifugation at $4^{\circ} \mathrm{C}(30$ $\mathrm{min} / 13,000 \mathrm{~g}$ ) the supernatant was collected and used as STAg. pTAg was obtained by immunoaffinity, using the N strain STAg and an affinity column of the anti-P32 mAb (Elsaid et al. 1999) coupled to cyanogen bromide-activated Sepharose 4B.

Liposome preparation and antigen incorporation - Antigens were incorporated into liposomes as described by Elsaid et al. (1999), using the dehydration-rehydration method (Kirby \& Gregoriadis 1984). Antigens-containing vesicles were washed three times in PBS at 13,000 $\mathrm{g}$ and the entrapped protein concentration was determined by the method described by Bradford (1976).

Immunization and challenge of Balb/c mice Female Balb/c mice were immunized subcutaneously with three inoculations at two-week intervals using a different type of antigens of $T$. gondii as shown in Table I. Three control groups were inoculated with either PBS, FCA with PBS, or empty liposome (L). All pregnant mice were challenged with four cysts of $\mathrm{P}$ strain orally 14 days after the final immunization between the 10th and 14th day of pregnancy.

Congenital disease model - Immediately after the third immunization, three virgins Balb/c immunized mice were housed with one male for five days and were then housed separately to rear their pups. Pregnant dams were infected between the 10th and 14th day of pregnancy. Litters were fostered to uninfected lactating dams as soon as possible after birth to prevent the possibility of infection through suckling. The numbers of live and

TABLE I

Protocols of immunization of female Balb/c mice with different Toxoplasma gondii antigens

\begin{tabular}{lcccccr}
\hline $\begin{array}{l}\text { Weeks prior } \\
\text { to challenge }\end{array}$ & L/STAg & L/SCAg & L/STCAg & L/pTAg & STAg & FCA/STAg \\
\hline 6 & $40 \mu \mathrm{g}$ & 2000 cysts & $\begin{array}{c}1000 \text { cyst }+ \\
20 \mu g \text { STAg }\end{array}$ & $5 \mu \mathrm{g}$ & $40 \mu \mathrm{g}$ & $40 \mu \mathrm{g}$ \\
4 & $40 \mu \mathrm{g}$ & 2000 cysts & $\begin{array}{c}1000 \mathrm{cyst}+ \\
20 \mu \mathrm{g} \mathrm{STAg}\end{array}$ & $5 \mu \mathrm{g}$ & $40 \mu \mathrm{g}$ & $40 \mu \mathrm{g}$ \\
2 & $40 \mu \mathrm{g}$ & 2000 cysts & $\begin{array}{c}1000 \mathrm{cyst}+ \\
20 \mu \mathrm{STAg}\end{array}$ & $5 \mu \mathrm{g}$ & $40 \mu \mathrm{g}$ & $40 \mu \mathrm{g}$ \\
\hline
\end{tabular}

L/STAg: soluble tachyzoite antigen in liposomes; L/SCAg: soluble tissue cyst antigen in liposomes; L/STCAg: tachyzoite plus tissue cyst soluble antigens in liposomes; L/pTAg: purified tachyzoite antigen in liposomes; STAg: soluble tachyzoite antigen; FCA/STAg: soluble tachyzoite antigen emulsified with Freund's complete adjuvant. 
dead pups were recorded. All surviving pups were killed by exsanguination under anesthesia at six weeks old. Detection of congenital infection was carried out according to Fux et al. (2000) through examination of cysts in the brain of the pups, by enzyme linked immunosorbent assay (ELISA) or bioassay of pup tissues. Pups were considered uninfected when antibodies to $T$. gondii were not found and when $T$. gondii parasites were not demonstrated.

ELISA - ELISA was performed as described by Fux et al. (2000). Briefly, microplate were coated with STAg. Sera were diluted 1:64 in PBStween-20 at $0.05 \%$, in duplicate and incubated at $37^{\circ} \mathrm{C}$ for $45 \mathrm{~min}$. Then the plate was washed and $100 \mu \mathrm{l}$ of peroxidase conjugated IgG mouse antiimmunoglobulin was added to each well. The reaction was visualized with orto-phenylenodiamine and stopped with $4 \mathrm{~N}-\mathrm{H}_{2} \mathrm{SO}_{4}$. Absorbance was read at $490 \mathrm{~nm}$ on a Microplate Reader BIORAD 3550.

Bioassay - The bioassay of pup tissues was carried out on newborn offspring, which died during the experimental period. Portions of the brain, heart, lung and liver of each dead pup were pooled, ground in a mortar with a pestle, suspended in antibiotic saline, and inoculated intraperitoneally into Swiss female. These mice were then examined for $T$. gondii infection after 30 days by parasitological and serologic tests (Fux et al. 2000).

Immune response in immunized mice - All mice were bled prior to immunization and seven days after the third immunization. Antibodies were determined by ELISA as described for detection of congenital infection.

T-cell proliferation assay - Spleens from three immunized mice with L/STAg, L/STCAg, L/pTAg,
STAg, FCA/STAg and PBS were removed aseptically. Splenocytes were cultured at a concentration of $2.5 \times 10^{5}$ cells $/ 0.1 \mathrm{ml}$ in RPMI 1640 , supplemented with $25 \mathrm{mM}$ sodium bicarbonate, 25 mM HEPES buffer (Sigma), 3mM L-glutamine, $10 \%$ of antibiotics and antimycotics $(10.000 \mathrm{U} / \mathrm{ml}$ penicillin, $10 \mathrm{mg} / \mathrm{ml}$ streptomycin and $25 \mu \mathrm{am}$ photericin B, Sigma) and 10\% fetal bovine serum. Cells were stimulated with $3.5 \mu \mathrm{g} / \mathrm{ml} \mathrm{STAg}$ or Con A in triplicate. The cells were incubated at $37^{\circ} \mathrm{C}$ in $5 \% \mathrm{CO}_{2}$, for three days. On the fourth day, 0.5 $\mu \mathrm{Ci}$ of $[3 \mathrm{H}]$ thymidine (NEN Research products, DuPont, Boston, US, sp. Act.6.7 Ci/nmol) was added to each well. After $6 \mathrm{~h}$, the cells were collected on glass filter strips and the mean count per minute (cpm) was determined by scintillation counting. The stimulation index (SI) was calculated as the mean cpm of antigen stimulated cultures divided by the mean cpm of unstimulated cultures. The base line level of positive was an SI $\geq 2.5$.

Statistical analysis - The incidence of congenital transmission, fetal death and SI were evaluated by the Chi-squared test and Student's $t$ test. The absorbance means of ELISA were examined using the Duncan test (Duncan 1955) at 5\% level of probability.

\section{RESULTS}

Vertical transmission - Congenital transmission occurred in all groups of immunized and non-immunized dams (Table II). The highest rates of congenital infection $(91 \%, 93 \%$ and $88 \%)$ were found in pups born from non-immunized dams (L/PBS, FCA/PBS and PBS). Only groups immunized with antigen-containing liposomes (L/STAg, L/SCAg,

TABLE II

Incidence of vertical disease infection and fetal death in Balb/c mice immunized with different Toxoplasma gondii antigens

\begin{tabular}{|c|c|c|c|c|c|c|}
\hline \multirow{2}{*}{\multicolumn{2}{|c|}{ Dams }} & \multirow{3}{*}{ Offspring } & \multicolumn{4}{|c|}{ Pups } \\
\hline & & & \multicolumn{2}{|c|}{ Deaths } & \multicolumn{2}{|c|}{ Infected } \\
\hline Groups & Number & & Number & $\%$ & Number & $\%$ \\
\hline L/STAg & 7 & 37 & 3 & $8 \%$ & 20 & $54 \%$ \\
\hline L/SCAg & 5 & 24 & 8 & $33 \%$ & 14 & $58 \%$ \\
\hline L/STCAg & 6 & 30 & 2 & $7 \%$ & 12 & $40 \%$ \\
\hline $\mathrm{L} / \mathrm{pTAg}$ & 6 & 38 & 2 & $5 \%$ & 15 & $39 \%$ \\
\hline STAg & 6 & 29 & 15 & $52 \%$ & 23 & $79 \%$ \\
\hline FCA/STAg & 6 & 27 & 10 & $37 \%$ & 24 & $89 \%$ \\
\hline FCA/PBS & 5 & 28 & 12 & $43 \%$ & 26 & $93 \%$ \\
\hline L/PBS & 5 & 22 & 10 & $45 \%$ & 20 & $91 \%$ \\
\hline PBS & 6 & 24 & 11 & $46 \%$ & 21 & $87.5 \%$ \\
\hline
\end{tabular}

L/STAg: soluble tachyzoite antigen in liposomes; L/SCAg: soluble tissue cyst antigen in liposomes; L/STCAg: tachyzoite plus tissue cyst soluble antigens in liposomes; L/pTAg: purified tachyzoite antigen in liposomes; STAg: soluble tachyzoite antigen; FCA/STAg: soluble tachyzoite antigen emulsified with Freund's complete adjuvant; FCA/PBS: Freund's complete adjuvant with PBS; L/PBS: liposomes with PBS; PBS: saline alone (control) 
L/STCAg and L/pTAg) showed a significant reduction in congenital transmission (40\% to 58\%) with respect to control groups $(p<0.05)$. It was also noteworthy that these groups showed a significantly lower level of transmission than the FCA/ STAg group $(p<0.05)$.

Pup mortality - The number of pups that were born dead or died after birth, was also determined (Table II). The highest mortality levels (43\%, 45\% and $46 \%$ ) were found in pup groups born of nonimmunized dams (FCA/PBS, L/PBS and PBS) or dams immunized with STAg in PBS alone (52\%). A much lower mortality rate $(8 \%, 7 \%$ and $5 \%)$ was found in pup groups originated from dams immunized with L/STAg, L/STCAg and L/pTAg. The latter pup group showed significantly lower mortality rate than all other groups $(p<0,05)$.

Immune response - Immunization of $\mathrm{Balb} / \mathrm{c}$ female mice with different $T$. gondii antigens resulted in significant antibody production (Table III) when compared with non-immunized control groups. Splenic cells of all mice immunized with the different preparations of antigen displayed significant $T$. gondii-specific proliferative responses following in vitro stimulation in the presence of STAg (Figure). Mice immunized with L/STAg, L/STCAg and L/pTAg presented higher stimulation index than L/SCAg and FCA/STAg, but differences were not statistically significant.

\section{DISCUSSION}

We describe here a protective immunity that limited congenital infection in an experimentallyinfected murine model. The important feature in this immunization is the use of a purified antigen of tachyzoites and a cocktail of life-cycle stage=
TABLE III

Optical density (OD) by ELISA of female Balb/c mice sera before immunization and after the third dose immunization

\begin{tabular}{lcc}
\hline Mice group & $\begin{array}{c}\text { OD } \pm \text { SD } \\
\text { before immunization }\end{array}$ & $\begin{array}{c}\text { OD } \pm \text { SD } \\
\text { after immunization }\end{array}$ \\
\hline L/STAg & $0.025 \pm 0.014$ & $0.220 \pm 0.050$ \\
L/SCAg & $0.016 \pm 0.011$ & $0.193 \pm 0.038$ \\
L/STCAg & $0.011 \pm 0.010$ & $0.279 \pm 0.068$ \\
L/pTAg & $0.025 \pm 0.016$ & $0.205 \pm 0.024$ \\
STAg & $0.009 \pm 0.009$ & $0.238 \pm 0.083$ \\
FCA/STAg & $0.019 \pm 0.011$ & $0.237 \pm 0.053$ \\
FCA/PBS & $0.014 \pm 0.008$ & $0.024 \pm 0.007$ \\
L/PBS & $0.015 \pm 0.016$ & $0.031 \pm 0.028$ \\
PBS & $0.017 \pm 0.009$ & $0.028 \pm 0.012$ \\
\hline
\end{tabular}

L/STAg: soluble tachyzoite antigen in liposomes; L/SCAg: soluble tissue cyst antigen in liposomes; L/STCAg: tachyzoite plus tissue cyst soluble antigens in liposomes; L/pTAg: purified tachyzoite antigen in liposomes; STAg: soluble tachyzoite antigen; FCA/ STAg: soluble tachyzoite antigen emulsified with Freund's complete adjuvant; FCA/PBS: Freund's complete adjuvant with PBS; L/PBS: liposomes with PBS; PBS: saline alone (control)

specific antigen (tachyzoites and tissue cysts). While previous studies in mice have indicated that vaccination with live-attenuated (McLeod et al. 1988) or STAg T. gondii in liposomes (Roberts et al. 1994) can reduce abortion and congenital toxoplasmosis transmission, this is the first time that a pTAg and a cocktail of life-cycle stage-specific antigen has been shown to protect against congenital infection.

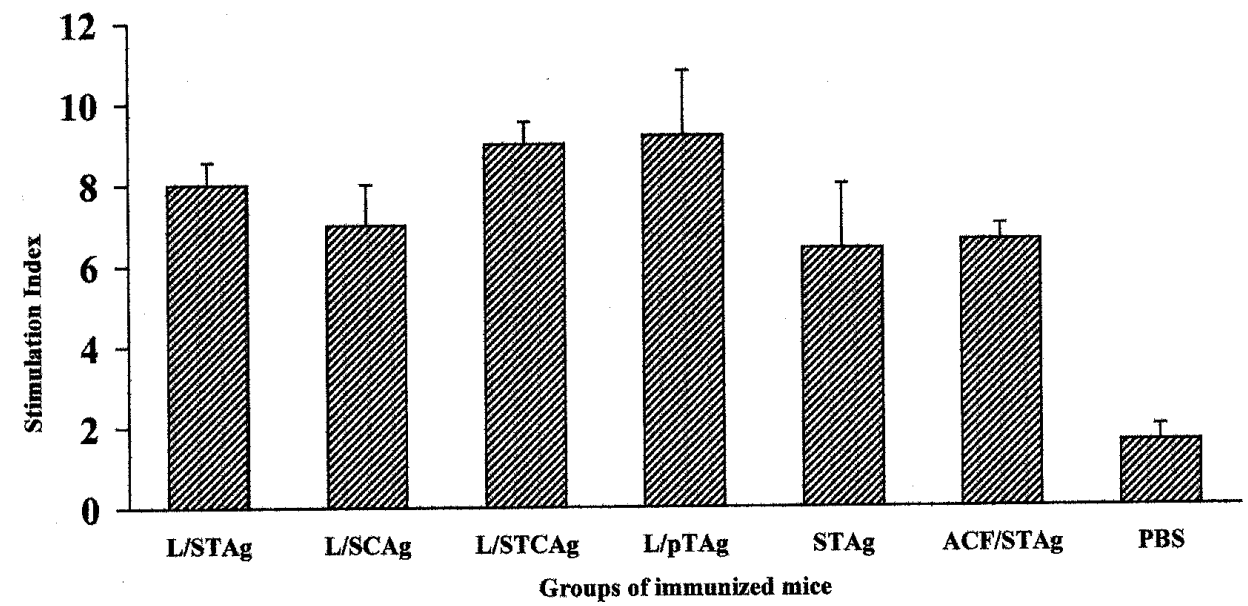

Mean of proliferative responses of the splenic cells of Balb/c mice immunized with $40 \mu \mathrm{g}$ of soluble tachyzoite antigen in liposomes (L/STAg), soluble tissue cyst antigen in liposomes (L/SCAg), tachyzoite plus tissue cyst soluble antigens in liposomes (L/STCAg), soluble tachyzoite antigen (STAg), soluble tachyzoite antigen emulsified with Freund's complete adjuvant (FCA/STAg) and $5 \mu \mathrm{g}$ of purified tachyzoite antigen in liposomes ( $/ \mathrm{pTAg}$ ), stimulated with $2.5 \mu \mathrm{g} / \mathrm{ml} \mathrm{STAg}$. PBS: saline alone (control) 
However, as demonstrated in previous studies by Roberts et al. (1994), Kasper et al. (1986), Bulow and Boothroyd (1991), Yap et al. (1998) and Khan et al. (1988), the adjuvant used can greatly influence the vaccine's effectiveness irrespective of the antigen involved. We found that the incorporation of TAg (purified P32 or soluble) in liposomes significantly increased the degree of protection against congenital transmission. The use of STAg emulsified in FCA or without adjuvant, did not show significant protection against vertical transmission. A similar result has been demonstrated by Roberts et al. (1994) using P30 emulsified in FCA.

As demonstrated by Roberts et al. (1994), vaccination with STAg without adjuvant increased the rate of fetal death, above that found in the litters from non-vaccinated dams. Our results confirm this observation. Immunization of Balb/c mice with STAg alone and subsequent infection with four cysts of the $\mathrm{P}$ strain result in the increased of the incidence of fetal death. Roberts et al. (1994) hypothesized that either STAg contained counter-protective as wells protective antigen, or that those antigens which are protective by one route (entrapped in vesicles) are exacerbative in the free soluble form. It is not known if the immunization of normal pregnant Balb/c mice using the described antigens, without parasite challenge infection, influence the lethality of the pups.

As we described previously (Elsaid et al. 1999), with the use of combining TAg and cysts encapsulated in liposomes, all mice survived after challenge with 80 cysts of $\mathrm{P}$ strain (LD90). Only 9 $(90 \%)$ and $7(70 \%)$ of the mice immunized with tachyzoite or cysts antigens, respectively, survived. In this study we compared the effect on vaccine efficacy of combining antigens of different stages to reduce congenital infection. Although the combination of both antigens (L/STCAg) reduced congenital transmission, the differences were not statistically significant when compared to the two other groups (L/STAg and L/SCAg).

The methods used here for the determination of infection are sensitive enough to detect congenital toxoplasmosis in Balb/c mice. Roberts and Alexander (1992), that proposed the present model, and Roberts et al. (1994), who tested vaccines in this model, just use ELISA to detect fetal infection. We evaluated congenital toxoplasmosis through ELISA, examination for cysts in the brain of the pups and bioassay of pup tissues (Fux et al. 2000). The association of these methods certainly increased the sensitivity of infection detection.

The antibody level by ELISA, after immunization, was significantly higher in all the groups of mice immunized with $T$. gondii antigen but the dif- ferences among groups were not statistically significant. In the same way, the proliferation of splenic cells happened in all the groups of immunized mice. While immunization with L/STAg, L/STCAg and $\mathrm{L} / \mathrm{pTAg}$ resulted in a spleen population, which produced higher specific proliferative responses than STAg emulsified in FCA or STAg in PBS, these differences were not significant. This seems to indicate that immunization with $\mathrm{L} / \mathrm{pTAg}$ or total tachyzoite and/or bradyzoite antigen incorporated into liposomes developed a protective immunity (humoral and cellular) that could contribute to the limitation of the systemic spread of $T$. gondii and reduce vertical disease transmission.

Our results, using a purified antigen or a cocktail of $T$. gondii antigen, confirm our previous study using an acute model of lethal murine toxoplasmosis (Elsaid et al. 1999) and support the findings of Roberts et al. (1994) who demonstrated prevention of vertical toxoplasmosis and fetal death by vaccination with STAg entrapped within non-ionic surfactant. It is worth investigating the protective potentialities of the P32 antigen and further with an antigen specific to the bradyzoite stage of the parasite in order to enhance protective immunity.

\section{ACKNOWLEDGEMENT}

To Dr Paul Williams for english review and Rosalida EN Lopes for technical assistance.

\section{REFERENCES}

Alexander J, Roberts CW, Brewer JM 1993. Progress towards the development of a vaccine against congenital toxoplasmosis: identification of protective antigens and the selection of the appropriate adjuvants. In JE Smith, Toxoplasmosis, NATO ASI Series H, vol. H 78, Elsevier Publications, Cambridge, p. 217-229.

Beverley JKA, Watson W 1971. Prevention of experimental and of naturally occurring ovine abortion due to toxoplasmosis. Vet Rec 88: 39-41.

Bradford MM 1976. A rapid and sensitive method for the quantitation of microgram quantities of protein utilizing the principle of protein dye binding. Anal Biochem 72: 248-254.

Bulow R, Boothroyd JC 1991. Protection of mice from fatal Toxoplasma gondii infection by immunization with P30 antigen in liposomes. J Immunol 147: 246251.

Duncan DB 1955. Multiple Range and Multiple F tests. Biometrics 11: 1-42.

Elsaid MMA, Vitor RWA, Frézard FJG, Martins MS 1999. Protection against toxoplasmosis in mice immunized with different antigens of Toxoplasma gondii incorporated into liposomes. Mem Inst Oswaldo Cruz 94: 485-490.

Fux B, Ferreira AM, Cassali GD, Tafuri WL, Vitor RWA 2000. Experimental toxoplasmosis in BALB/c mice. Prevention of vertical disease transmission by treat- 
ment and reproductive failure in chronic infection. Mem Inst Oswaldo Cruz 95: 121-126.

Holland D, Bretsher P, Russel A 1984. Immunologic and inflammatory responses during pregnancy. $J$ Clin Lab Immunol 14: 177-179.

Jamra LMF, Vieira MPL 1991. Isolamento do Toxoplasma gondii de exsudato peritoneal e órgãos de camundongos com infecção experimental. Rev Inst Med Trop São Paulo 133: 435-441.

Kasper LH, Currie KM, Bradley MS 1986. An unexpected response to vaccination with a purified major membrane tachyzoites antigen (P30) of Toxoplasma gondii. J Immunol 134: 3426-3432.

Khan IA, Smith JS, Kasper LH 1988. Induction of antigen-specific parasiticidal cytotoxic T cell splenocytes by a major membrane protein (P30) of Toxoplasma gondii. J Immunol 141: 3600-3606.

Kirby C, Gregoriadis G 1984. Dehydration rehydration vesicles: a simple method for high yield drug entrapment in liposomes. Biotechnology 2: 979-984.

Luft BJ, Hafner MD, Korzun AH, Leport C, Antoniskis DEM, Boster DD, Bourland R, Ultamchndani R, Fuhrer J, Jacobson J, Morlat P, Vilde J, Remington JS 1993. Toxoplasmic encephalitis in patients with the acquired immunodeficiency syndrome. $N$ Engl J Med 329: 995-1000.

McLeod R, Frenkel JR, Estes RG, Mack DG, Eisenhauer PB, Gibbons G 1988. Subcutaneous and intestinal vaccination with tachyzoites of Toxoplasma gondii and acquisition of immunity to peroral and congenital toxoplasma challenge. J Immunol 140: 1632-1637.

Nobrega P, Trapp E, Giovannoni M 1952. Toxoplasmose epizótica em coelhos. I. Ação da sulfadiazina. Ciênc Cult 4: 134-135.
Petersen E, Nielsen HV, Christiansen L, Spenter J 1998. Immunization with $E$. coli produced recombinant $T$. gondii SAG1 with alum as adjuvant protect mice against lethal infection with Toxoplasma gondii. Vaccine 16: 1283-1289.

Remington JS, Mcleod R, Desmonts G 1994. Toxoplasmosis. In JS Remington, OJ Klein (eds), Infectious Disease of the Fetus and New-born Infant, WB Saunders Co, Philadelphia, p. 141-267.

Roberts CW, Alexander J 1992. Studies on a murine model of congenital toxoplasmosis: vertical disease transmission only occurs in BALB/c mice infected for the first time during pregnancy. Parasitology 104: 19-23.

Roberts CW, Brewer JM, Alexander J 1994. Congenital toxoplasmosis in Balb/c mouse: prevention of vertical disease transmission and fetal death by vaccination. Vaccine 12: 1389-1394.

Thouvenin M, Candolfi E, Villard O, Klein JP, Kien T 1997. Immune response in a murine model of congenital toxoplasmosis: increased susceptibility of pregnant mice and transplacental passage of Toxoplasma gondii are Type 2-dependent. Parasitology 39: 279-283.

Wegmann TG, Lin A, Guilbert L, Mosmann TM 1993. Bidirectional cytokine interactions in the maternalfetal relationship: is successful pregnancy a Th2 phenomenon? Immunol Today 14: 353-356.

Wong SY, Remington JS 1994. Toxoplasmosis in pregnancy. Clin Infect Dis 18: 853-862.

Yap GS, Scharton-kersten T, Ferguson DJP, Howe D, Suzuki Y, Sher A 1998. Partially protective vaccination permits the development of latency in a normally virulent strain of Toxoplasma gondii. Infect Immun 66: 4382-4388. 